Artigo original

\title{
Métodos de avaliação antropométrica e bioimpedância: um estudo correlacional em trabalhadores da indústria
}

\author{
Anthropometric and bioimpedance evaluation methods: a correlational study in \\ industrial workers
}

Analie Nunes Couto ${ }^{1}$, Kely Lisandra Dummel ${ }^{1}$, Jane Dagmar Pollo Renner ${ }^{1}$, Hildegard Hedwig Pohl' ${ }^{1}$.

${ }^{1}$ Universidade de Santa Cruz do Sul, Santa Cruz do Sul, RS, Brasil.

Submissão: 01/09/2016

Aceite: 16/09/2016

analiecouto@hotmail.com

\begin{abstract}
RESUMO
Justificativa e Objetivos: A avaliação do estado nutricional através da antropometria e bioimpedância elétrica (Bia) surge como uma ferramenta promissora de custo mais acessível. Este estudo verificou a correlação entre dois métodos de avaliação corporal (avaliação antropométrica e da composição corporal e área de gordura visceral por Bia) em trabalhadores da indústria. Métodos: Trata-se de um estudo transversal, de caráter descritivo e correlacional, com 22 trabalhadores industriais, do município de Santa Cruz do Sul-RS. Para a avaliação da composição corporal foram aferidas variáveis antropométricas: Índice de Massa Corporal (IMC), Circunferência da Cintura (CC), Risco Cintura Quadril (RCQ), percentual de gordura corporal $(\% \mathrm{G})$, peso de gordura (PG), massa corporal magra (MCM) e peso ósseo (PO). Para a composição corporal, utilizou-se a Bia, através do analisador de multi-frequência octopolar (InBody 720) para as variáveis: conteúdo mineral (CM); massa de gordura (MG); massa musculoesquelética (MME), IMC, \%G, RCQ e área de gordura visceral (AGV). Na análise estatística, utilizou-se o teste de correlação de Pearson ou Spearman para avaliar a correlação entre as variáveis. Resultados: Dos 22 trabalhadores, $72,7 \%$ eram do sexo feminino, com idade média de 37,73 anos. Apresentaram IMC médio de $26,14 \mathrm{~kg} / \mathrm{m}^{2}$, o que classifica a amostra com sobrepeso. No estudo, as avaliações feitas pelas duas técnicas apresentaram uma forte correlação entre as variáveis IMC e IMCBia, \%G e \%GBia, PG e MGBia, MCM e MMEBia, PO e CMBia, CC e AGVBia. Conclusão: Os dois métodos mostraram obter comportamento estatístico semelhante na avaliação corporal, sugerindo a possibilidade de utilização de quaisquer destas técnicas para avaliação da composição corporal.
\end{abstract}

DESCRITORES: Antropometria. Composição corporal. Impedância bioelétrica. Adiposidade central. Trabalhadores.

\begin{abstract}
Background and Objectives: The assessment of nutritional status by anthropometry and bioelectrical impedance analysis (Bia), emerges as a promising tool more affordable. This study examined the correlation between two body evaluation methods (anthropometric and body composition and visceral fat area by Bia) in industrial workers. Methods: This is a crosssectional, descriptive and correlational character, with 22 industrial workers, the city of Santa Cruz do Sul-RS. For the assessment of body composition were measured anthropometric variables: body mass index (BMI), waist circumference (WC), Risk Waist Hip ratio (WHR),
\end{abstract}


body fat percentage (BF\%), fat weight (PG); lean body mass (LBM); bone weight (PO). For body composition used to Bia through the octopolar multi-frequency analyzer (In-Body 720) for the variables: mineral content (CM); fat mass (MG); musculoskeletal mass (MME); BMI; $\%$ G; WHR and visceral fat area (VFA). In the statistical analysis we used the Pearson correlation test to evaluate the correlation between the variables. Results: Of the 22 workers, $72.7 \%$ were female, mean age of 37.73 years. They had an average BMI of $26,14 \mathrm{~kg} / \mathrm{m}^{2}$, which classifies the sample overweight. In the study, the assessments made by the two techniques showed a strong correlation between $\mathrm{BMI}$ and IMCBia variables\% $\mathrm{F}$ and\% GBia, $\mathrm{PG}$ and MGBia, MCM and MMEBia, PO and CMBia, DC and AGVBia. Conclusion: Both methods showed obtain similar statistical behavior in body evaluation, suggesting the possibility of using any of these techniques to assess body composition.

KEYWORDS: Anthropometry. Body composition. Bioelectrical impedance. Central adiposity. Workers.

\section{INTRODUÇÃO}

Diferentes aspectos podem incidir sobre a saúde dos trabalhadores, seja no ambiente fabril ou em outros setores da atividade produtiva, como as alterações no estado nutricional que possuem implicações na manutenção da saúde e na morbi-mortalidade, quando associada a múltiplos processos crônicos. ${ }^{1,2}$ As alterações da composição corporal implicam diretamente no estado nutricional e se detectadas e avaliadas o mais precocemente possível,contribuem na redução dos fatores de risco decorrentes dos problemas de saúde. ${ }^{3}$

Na avaliação do estado nutricional a utilização de métodos duplamente indiretos surge como uma promissora ferramenta. Para essa avaliação, podem ser utilizados diferentes métodos, como a pesagem hidrostática ou métodos por imagem, absortometria por dupla emissão de raios-X (DEXA), ressonância magnética e tomografia computadorizada (TC), considerados métodos de referência, mais acurados e bastante onerosos, contudo são de alto custo, exigem uma equipe especializada e uma estrutura física complexa. Em contrapartida, existem métodos de fácil execução e custo mais acessível, como a avaliação por antropometria e Impedância Bioelétrica ou Bioimpedância(Bia). ${ }^{3-5}$

A antropometria é definida como a ciência que estuda as mensurações da forma, tamanho e composição corporal humana, devido a facilidade na aplicação e melhor aceitação da populaçãoé o método de maior utilização na prática clínica e estudos epidemiológicos. ${ }^{6-8}$ Destaca-se o popular cálculo do índice de massa corporal (IMC) que é um dos indicadores antropométricos mais utilizados na identificação de risco nutricional, como também a mensuração das dobras cutâneas (DC), circunferência da cintura (CC), relação cintura-estatura (RCE), diâmetro abdominal sagital (DAS), índice de conicidade (IC) e relação cintura-quadril (RCQ)entre outros.No entanto, estas medidas antropométricas não são capazes de diagnosticar a gordura visceral separadamente da gordura abdominal subcutânea. 5,7,9 
Atualmente, a Biatem sido validada e utilizada para estimar a composição corporal e o estado nutricional de indivíduos não somente saudáveis, mas também em diversas situações clínicas. É um método indireto utilizado para avaliar a composição corporal, que estima compartimentos de massa muscular, gordura, hídrico e também avalia a área de gordura visceral (AGV). Tem como base o princípio de que o fluxo da corrente elétrica possui taxas diferentes pelo corpo, de acordo com a sua composição, tendo o tecido muscular uma resistência menor à corrente elétrica do que o tecido adiposo. ${ }^{10,11}$

Tais técnicas de avaliação são largamente aceitas como seguras, rápidas e confiáveis capazes de avaliar o estado nutricional e a composição corporal dos indivíduos. Diante do exposto, o objetivo deste estudo foi verificar correlação entre dois métodos de avaliação corporal (avaliação antropométrica e da composição corporal e AGVpor Bia em trabalhadores da indústria.

\section{MÉTODOS}

Trata-se de um estudo transversal, de caráter descritivo e correlacional. A amostra foi constituída de 22 trabalhadores industriais, do município de Santa Cruz do Sul-RS, que aceitaram participar das reavaliações efetuadas na pesquisa "Novas Abordagens em Biodinâmica para Diagnóstico e Prevenção de Obesidade e Comorbidades em Trabalhadores e Escolares", aprovada pelo Comitê de Ética e Pesquisa com Seres Humanos sob protocolo no 703.934/14 e que assinaram o termo de consentimento livre e esclarecido.

Foram selecionadas variáveis demográficas, sexo e idade. Para a avaliação da composição corporal foram aferidas variáveis antropométricas [IMC, CC, RCQ, percentual de gordura corporal $(\% \mathrm{G})$, peso de gordura (PG); massa corporal magra (MCM); peso ósseo (PO)] e da composição corporal por Bia [conteúdo mineral (CM); massa de gordura (MG); massa musculoesquelética (MME); IMC; \%G; RCQ e AGV].

O peso e a estatura foram aferidos utilizando a balança antropométrica (Welmy SA, Santa Bárbara do Oeste, Brasil). Calculou-se o IMC, obtido através da medida do peso corporal (Kg), dividido pela estatura $(\mathrm{m})$ elevada ao quadrado $\left(\mathrm{P} / \mathrm{E}^{2}\right)$, categorizado conforme os parâmetros da Organização Mundial da Saúde. ${ }^{12}$

As DC (peitoral, tricipital, subescapular, suprailíaca, abdominal, coxa e axilar média) foram mensuradas por meio de plicômetro científico de marca Lange ${ }^{\circledR}$ Skinfold Caliper (Beta Technology INC, Santa Cruz, CA, EUA), com pressão constante de $10 \mathrm{~g} / \mathrm{mm}^{2}$ na superfície de contato, precisão de $1 \mathrm{~mm}$ e escala de $0-65 \mathrm{~mm}$, com sensibilidade de $0,1 \mathrm{~mm}$, com 3 repetições. 
O \%G foi obtido através da soma das sete DC, sendo a densidade corporal calculada através da fórmula de Jackson e Pollocke posteriormente a equação de Siri. ${ }^{13}$ Utilizou-se fita métrica inelástica da marca Cardiomed, comprimento de $150 \mathrm{~cm}$, dividida em centímetros e subdividida em milímetros para as medidas de CC e circunferência do quadril (CQ), para estimativa da RCQ, mensurado, observando os critérios de Heyward. ${ }^{13}$

Para estimativa do $\mathrm{PG}$, utilizou-se a fórmula $\mathrm{PG}=$ peso $\mathrm{x}(\% \mathrm{G} / 100)$. Para o percentual de MCM utiliza-se a diferença entre o peso e o PG. Já o PO resulta da equação: 3,02 ( $\mathrm{H}^{2}$ x R x F x 400) 0,712 representando $\mathrm{o} \mathrm{H}^{2}$ a estatura em metros ao quadrado, $\mathrm{R}$ representa o diâmetro biestiloide rádio ulnar e $\mathrm{F}$ representa o diâmetro biepicondiliano do fêmur, ambos expressos em centímetros. $^{14}$

A composição corporal foi estimada por meio da Bia, utilizando-se o analisador de multifrequência octopolar (In-Body 720; Biospace, Seoul, Coreia do Sul). O InBody 720 usa 8 elétrodos, sendo dois em contato com a palma de cada mão (E1, E3) e o polegar (E2, E4) e dois em contato com a parte anterior (E5, E7) e posterior (E6, E8) da planta de cada pé. Este aparelho avalia cinco impedâncias segmentares (braço direito, braço esquerdo, perna direita, perna esquerda e tronco), medidas em seis frequências diferentes $(1,5,50,250,500$ e $1000 \mathrm{KHz}){ }^{11,15}$

Os pontos de contato do corpo com os eletrodos foram previamente limpos com um tecido eletrolítico. As avaliações seguiram o protocolo indicado pelo fabricante. Os participantes foram orientados a não consumir álcool 48 horas antes do teste; fazer 12 horas de jejum e não realizar exercício de intensidade moderada à elevada nas 12 horas antes da avaliação; não realizar o teste perante a presença de um estado febril ou de desidratação; ir ao banheiro antes do teste; usar roupas leves e remover joias e objetos metálicos ou implantes dentários com metal (quando possíveis de serem removidos) e não ingerir café antes do início dos testes.

A análise estatística, realizada no programa SPSS versão 20.0, foi feita através de estatística descritiva, utilizando frequência, percentual, média e desvio padrão e correlacional, sendo primeiramente testada a normalidade dos dados e após aplicados os testes de correlação de Pearson ou Spearman. Os níveis de correlação foram classificados conforme Dancey e Reidy. $^{16}$

\section{RESULTADOS}

Participaram do estudo 22 trabalhadores, sendo 72,7\% do sexo feminino, com média de idade de 37,73 anos. Em relação às características antropométricas cabe ressaltar que o IMC 
médio foi $26,14 \mathrm{~kg} / \mathrm{m}^{2}$, o que classifica a amostra com sobrepeso. Entretanto, ao analisar o \%G, tanto os homens como as mulheres ficaram classificados como acima da média com percentuais de $17,07 \%$ e $26,61 \%$, respectivamente, o que difere do $\% \mathrm{G}$ observado pela Bia, que teve média geral de $30,11 \%$, que classifica os sujeitos com percentual de gordura alto (Tabela 1).

Observa-se, ainda, que nas variáveis de Bia, a AGV dos sujeitos ficou abaixo dos valores de risco, com média de $83,75 \mathrm{~cm}^{2}$, o que também pode ser visto na estimativa de risco cardiovascular estimado pela CC, que apresentou média de 79,84 cm, apontando estarem sem risco ou classificados como adequados.

Tabela 1 - Caracterização geral e composição corporal da amostra através de variáveis antropométricas e de bioimpedância.

\begin{tabular}{|c|c|c|c|}
\hline \multirow[b]{2}{*}{ Variáveis } & \multicolumn{2}{|c|}{ Sexo } & \multirow[b]{2}{*}{$\begin{array}{c}\text { Geral } \\
22(100 \%)\end{array}$} \\
\hline & $\begin{array}{c}\text { Feminino } \\
16(72,7 \%)\end{array}$ & $\begin{array}{c}\text { Masculino } \\
6(27,3 \%)\end{array}$ & \\
\hline Idade(anos) & $37,25 \pm 6,35$ & $39,00 \pm 3,40$ & $37,73 \pm 5,68$ \\
\hline \multicolumn{4}{|l|}{ Antropometria } \\
\hline $\mathrm{IMC}\left(\mathrm{kg} / \mathrm{m}^{2}\right)$ & $26,37 \pm 5,77$ & $25,52 \pm 2,00$ & $26,14 \pm 4,98$ \\
\hline $\mathrm{CC}(\mathrm{cm})$ & $78,08 \pm 11,86$ & $84,5 \pm 6,45$ & $79,84 \pm 10,91$ \\
\hline RCQ & $0,77 \pm 0,06$ & $0,86 \pm 0,03$ & $0,80 \pm 0,07$ \\
\hline$\% \mathrm{G}$ & $26,61 \pm 6,13$ & $17,07 \pm 4,25$ & $23,99 \pm 7,09$ \\
\hline PG $(\mathrm{kg})$ & $17,42 \pm 7,86$ & $13,08 \pm 4,30$ & $16,24 \pm 7,24$ \\
\hline $\operatorname{MCM}(\mathrm{kg})$ & $45,61 \pm 5,61$ & $62,81 \pm 10,45$ & $50,30 \pm 10,49$ \\
\hline PO $(\mathrm{kg})$ & $8,26 \pm 0,83$ & $12,07 \pm 3,02$ & $9,30 \pm 2,38$ \\
\hline \multicolumn{4}{|l|}{ Bioimpedância } \\
\hline $\mathrm{CM}(\mathrm{kg})$ & $2,78 \pm 0,30$ & $4,00 \pm 0,86$ & $3,11 \pm 0,74$ \\
\hline MG (kg) & $22,20 \pm 11,46$ & $15,98 \pm 4,41$ & $20,50 \pm 10,32$ \\
\hline MME (kg) & $22,49 \pm 2,41$ & $33,82 \pm 6,41$ & $25,58 \pm 6,37$ \\
\hline $\operatorname{IMC}\left(\mathrm{kg} / \mathrm{m}^{2}\right)$ & $26,29 \pm 6,02$ & $25,45 \pm 1,98$ & $26,06 \pm 5,19$ \\
\hline$\% \mathrm{G}$ & $33,50 \pm 10,56$ & $21,07 \pm 4,45$ & $30,11 \pm 10,79$ \\
\hline RCQ & $0,95 \pm 0,06$ & $0,92 \pm 0,05$ & $0,94 \pm 0,06$ \\
\hline $\mathrm{AGV}\left(\mathrm{cm}^{2}\right)$ & $88,29 \pm 38,42$ & $71,63 \pm 21,83$ & $83,75 \pm 35,01$ \\
\hline
\end{tabular}

IMC: índice de massa corporal; CC: circunferência da cintura; RCQ: relação cintura-quadril; \%G: percentual de gordura; PG: peso de gordura; MCM: massa corporal magra; PO: peso ósseo; CM: conteúdo mineral; MG: massa de gordura; MME: massa músculo-esquelética; AGV: área de gordura visceral.

Ao correlacionar as variáveis de composição corporal estimadas pela antropometria com aquelas estimadas pela Bia (Tabela 2), nota-se que as variáveis IMC versus IMC Bia, \%G versus \%GBia, PG versus MGBia, MCM versus MMEBia, PO versus CMBia e CC versus AGVBia apresentaram uma forte correlação, com diferença significativa estatisticamente, demonstrando que as avaliações feitas pelas diferentes técnicas tem um comportamento semelhante, o que indica a possibilidade de utilizar quaisquer destas técnicas para avaliação da composição corporal no presente grupo.

Tabela 2 - Correlação entre composição corporal por antropometria e por bioimpedância. Bioimpedância

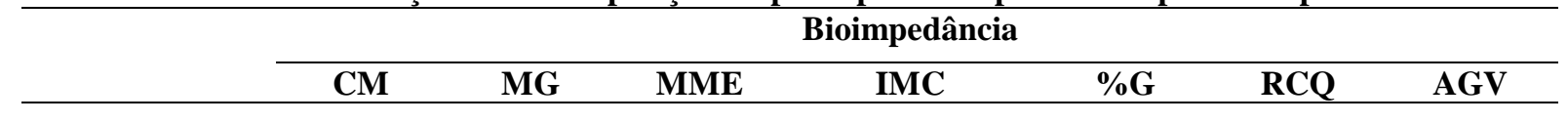




\begin{tabular}{lccccccc}
\hline Antropometria & & & & & & & \\
IMC & $0,151^{\dagger}$ & $0,853^{* \dagger}$ & $0,177^{\dagger}$ & $0,979^{* \dagger}$ & $0,619^{* \dagger}$ & $0,663^{\dagger}$ & $0,853^{* \dagger}$ \\
CC & $0,415^{\dagger}$ & $0,665^{* \dagger}$ & $0,428^{* \dagger}$ & $0,843^{* \dagger}$ & $0,528^{*+\dagger}$ & $0,540^{* \dagger}$ & $0,781^{*+\dagger}$ \\
RCQ & $0,559^{* \dagger}$ & $0,284^{\dagger}$ & $0,610^{* \dagger}$ & $0,549^{* \dagger}$ & $0,069^{+\dagger}$ & $0,227^{\dagger}$ & $0,345^{+\dagger}$ \\
$\mathbf{\% G}$ & $-0,442^{* \dagger}$ & $0,791^{* \dagger}$ & $-0,482^{* \dagger}$ & $0,601^{* \dagger}$ & $0,907^{*+}$ & $0,763^{* \dagger}$ & $0,839^{*+\dagger}$ \\
PG & $0,011^{\dagger}$ & $0,939^{* \dagger}$ & $-0,003^{\dagger}$ & $0,870^{* \dagger}$ & $0,802^{* \dagger}$ & $0,870^{* \dagger}$ & $0,951^{* \dagger}$ \\
MCM & $0,819^{* \dagger}$ & $0,312^{\dagger}$ & $0,835^{* \dagger}$ & $0,522^{* \dagger}$ & $-0,100^{\dagger}$ & $0,306^{\dagger}$ & $0,363^{\dagger}$ \\
PO & $0,923^{* \dagger}$ & $-0,021^{\dagger}$ & $0,891^{*+}$ & $0,136^{\dagger}$ & $-0,386^{+}$ & $0,121^{\dagger}$ & $0,042^{\dagger}$ \\
\hline
\end{tabular}

IMC: índice de massa corporal; CC: circunferência da cintura; RCQ: relação cintura-quadril; \%G: percentual de gordura; PG: peso de gordura; MCM: massa corporal magra; PO: peso ósseo; CM: conteúdo mineral; MG: massa de gordura; MME: massa músculo-esquelética; AGV: área de gordura visceral; *: p<0,05; ${ }^{\dagger}$ : correlação de Spearman; ${ }^{\dagger+}$ : correlação de Pearson.

\section{DISCUSSÃO}

Este estudo verificou a correlação entre dois métodos de avaliação corporal, utilizando a antropometria e a estimativa da composição corporal e AGV por Bia elétrica em trabalhadores da indústria, como meios de investigação de discrepância entre os métodos. No estudo, as avaliações feitas pelas duas técnicas tiveram um comportamento semelhante, apresentando uma forte correlação entre as variáveis IMC e IMCBia, \%G e \%GBia, PG e MGBia, MCM e MMEBia, PO e CMBia, CC e AGVBia, indicando a possibilidade de utilizar quaisquer destas técnicas para avaliação da composição corporal.

Corroborando os resultados apresentados, Fett et al., ao compararem diferentes métodos de avaliação da composição corporal em mulheres com sobrepeso e obesas sedentárias, submetidas a dois meses de treinamento em circuito ou caminhada, também observaram que as médias da percentagem de gordura da Bia e da antropometria não diferiam entre si e eram significativamente correlacionadas. ${ }^{17}$

Machado, Coelho e Coelho ao comparar e avaliar o grau de concordância dos percentuais de gordura corporal em idosos por três diferentes métodos: pela área adiposa do braço, pela prega cutânea tricipital (PCT) e por Bia, observaram que todos percentuais de gordura apresentaram associação estatisticamente significativa com as variáveis antropométricas. Segundo os autores, o \%GBia apresentou melhor correlação com o IMC, que reflete a massa corporal total e com a $\mathrm{CC}$, que reflete o depósito abdominal e, ainda, que o \%GBia teve correlação boa com o percentual de gordura estimado pela PCT, através da equação de Siri (\%GSiri), com significância estatística. ${ }^{1}$

Da mesma maneira, esta pesquisa encontrou resultados semelhantes, pois o IMC, o \% G e a CC por antropometria apresentaram uma forte correlação com a MGBia, com o IMCBia e com a AGVBia para ambas avaliações. Martins et al. observaram correlação entre os valores 
do \%G obtido pelo somatório das DC e pela Bia, ao compararem estes dois métodos de avaliação, evidenciando associação moderada entre estes. ${ }^{3}$ Já, Andrade Jr., Clemente e Gomes identificaram correlação entre a Gordura Corporal e CC. ${ }^{18}$

Rezende et al. com o objetivo de verificar a eficiência do IMC em identificar indivíduos com excesso de gordura corporal e com obesidade abdominal, constataram que a CC foi a medida antropométrica que mais se correlacionou com o IMC e com o \%G estimado por Bia. ${ }^{19}$ Segundo os autores, o IMC apresentou alta sensibilidade para diagnosticar indivíduos com \% G e CC, entretanto, na avaliação individual, o IMC não foi adequado para esse mesmo diagnóstico, devido aos baixos valores preditivos positivos encontrados, confirmando a necessidade de utilizar outras medidas antropométricas ou da composição corporal na avaliação do estado nutricional.

$\mathrm{Na}$ classificação do estado nutricional, a adoção do IMC como padrão único pode ocasionar avaliações equívocas, por estimar somente a relação entre o peso e a altura dos indivíduos, podendo proporcionar um diagnóstico incorreto. O IMC pode apresentar limitações em situações como: a relação com a proporcionalidade do corpo, ou seja, indivíduos com pernas curtas terão IMC aumentado; não considera a relação de massa livre de gordura e o desenvolvimento muscular, podendo levar a interpretações equivocadas na identificação da obesidade; a correlação com a estatura que, apesar de baixa, pode ser significativa, em crianças e adolescentes e em idosos a perda da massa muscular (sarcopenia) e o acúmulo abdominal de gordura.Com o envelhecimento, ocorre aumento de gordura corporal, principalmente na região abdominal, o que pode tornar a utilização do IMC cada vez mais limitada para a avaliação do estado nutricional nesta população, uma vez que indivíduos eutróficos e com sobrepeso também podem apresentar risco de alterações metabólicas. ${ }^{19,20}$

Neves et al. encontraram maior correlação entre análise por ultrassom e a Bia $(0,767)$, do que pela Bia e a avaliação da composição corporal pelas técnicas de DC para estimar o \%G $(0,742)$ e ultrassom com as DC $(0,709)$ em adultos jovens. ${ }^{21}$ Identificaram uma diminuição nas correlações obtidas na comparação entre todos os métodos, em sujeitos com IMC maior ou igual a 25 e, que estes resultados parecem reforçar a limitação da estimativa da avaliação corporal em sujeitos mais obesos.

Nobuyuki et al. avaliaram a confiabilidade e validade da Bia de multi-frequência octopolar desenvolvido e disponibilizado na Coréia (In-Body 720; Biospace, Seoul, Coréia do Sul) para a determinação da composição corporal japonesa. ${ }^{22}$ Comparando os resultados do InBody720 com aqueles obtidos pela DEXA, encontraram uma clara correlação entre os resultados de porcentagem de gordura corporal, massa músculo esquelética, $\mathrm{MCM}$ e CM. 
Ling et al. em seu estudo com 484 indivíduos de meia-idade participantes do Estudo de Longevidade, na cidade de Leiden na Holanda, examinaram a precisão da Bia segmentar de multifrequência (In-Body 720) na avaliação de diferentes parâmetros de composição corporal, utilizando DEXA como padrão de referência. ${ }^{15}$ Os pesquisadores concluíram que a Bia é uma ferramenta válida para as avaliações de composição corporal total e do corpo segmentar na população geral de meia-idade, particularmente para a quantificação da massa magra corporal.

O modelo da Bia segmentar (In-Body 720) inclui a avaliação da AGV, por meio da análise isolada do tronco estimando com mais precisão essa gordura. ${ }^{10}$ Segundo Leeet al. o método Bia pode ser considerado seguro e conveniente para medir a AGV em comparação com a TC tradicional. ${ }^{23} \mathrm{~A}$ Bia tem sido considerada suficientemente válida e segura. ${ }^{24}$

Pode-se considerar que, embora tenham sido utilizados somente dois métodos de avaliação, a não utilização de um método considerado padrão-ouro, como a DEXA ou a TC considerados métodos de referência, justifica-se devido a estudos nacionais e internacionais indicarem a utilização da Bia elétrica de multifrequência (InBody720), como um método altamente confiável e válido para determinar a composição corporal e AGV, e que a precisão do método Bia é bem estabelecida. Acrescido a isso, como uma limitação deste estudo pontuase, o número de elementos da amostra. ${ }^{3,4,15,22,25}$

O presente estudo permitiu concluir que os dois métodos mostraram comportamento semelhante na avaliação corporal, apresentando uma forte correlação entre as variáveis de estimativa da gordura corporal e abdominal/visceral, de massa magra e ósseo/mineral por antropometria e Bia, sugerindo a possibilidade de utilização de quaisquer destas técnicas para avaliação da composição corporal. As medições precisas de avaliação corporal são fundamentais para a identificação de alterações da composição corporal e suas implicações no estado nutricional em várias circunstâncias clínicas. Assim, por serem métodos mais simples, baratos, práticos e confiáveis, quando comparados ao padrão-ouro, estes dois métodos de avaliações podem ser consideradas ferramentas de rastreio simples para a caracterização de risco,em indivíduos.

AGRADECIMENTOS: Secretaria da Ciência, Inovação e Desenvolvimento Tecnológico do Rio Grande do Sul, sob o processo número: 270-2500/11-5, e Coordenação de Aperfeiçoamento de Pessoal de Nível Superior (CAPES).

\section{REFERÊNCIAS}


1. Machado RSP, Coelho MASC, Coelho KSC. Percentual de gordura corporal em idosos: comparação entre os métodos de estimativa pela área adiposa do braço, pela dobra cutânea tricipital e por bioimpedância tetrapolar. Rev Bras Geriatr Gerontol 2010; 13(1): 17-27. DOI: http://dx.doi.org/10.1590/S1809-98232010000100003.

2. Araújo MPN, Costa-Souza J, Trad LAB. A alimentação do trabalhador no Brasil: um resgate da produção científica nacional. Hist Cienc Saúde 2010; 17(4): 975-992. DOI: http://dx.doi.org/10.1590/S0104-59702010000400008.

3. Martins KA, Monego ET, Paulinelli RR, et al. Comparação de métodos de avaliação da gordura corporal total e sua distribuição. Rev Bras Epidemiol 2011; 14(4): 677-687. DOI: http://dx.doi.org/10.1590/S1415-790X2011000400014.

4. Martins GQ, Matheus SC, Santos DL, et al. Comparison of anthropometric equations to estimate body fat in subjects with overweight. Nutr Clín Diet Hosp 2015; 35(3): 27-33. DOI: http://dx.doi.org/10.12873/353martinsguil.

5. Morais PAO, Dantas MGB, Pitangui ACR, et al. Métodos indiretos para mensuração da gordura corporal em adolescentes escolares. Rev Bras Promoc Saude 2013; 26(3): 412-418.

6. Matheus SC, Santos L, Behenck MS, et al. O uso da antropometria para avaliar a distribuição de gordura corporal de pacientes com HIV/AIDS. Arq Ciênc Saúde 2015; 22(1): 64-69.

7. Petribú MMV, Guimarães FJSP, Cabral PC, et al. Desenvolvimento e validação de equação preditiva da gordura visceral em mulheres jovens. Rev Bras Cineantropom Desempenho hum 2012; 14(3): 333-342. DOI: http://dx.doi.org/10.5007//1980-0037.2012v14n3p333.

8. Silva DAS, Pelegrini A, Pires-Neto CS, et al. O antropometrista na busca de dados mais confiáveis. Rev Bras Cineantropom Desempenho Hum 2011; 13(1): 82-85. DOI: http://dx.doi.org/10.5007/1980-0037.2011v13n1p82.

9. Tchernof A, Després JP. Pathophysiology of human visceral obesity: an update. Physiol Rev 2013; 93(1): 359-404. DOI: http://dx.doi.org/10.1152/physrev.00033.2011. 
10. Eickemberg M, Oliveira CC, Roriz AKC, Sampaio LR. Bioimpedância elétrica e sua aplicação em avaliação nutricional. Rev Nutr 2011; 24(6): 873-882. DOI:

http://dx.doi.org/10.1590/S1415-52732011000600009.

11. Eickemberg M, Oliveira CC, Roriz AKC, et al. Bioimpedância elétrica e gordura visceral: uma comparação com a tomografia computadorizada em adultos e idosos. Arq Bras Endocrinol Metab 2013; 57(1): 27-32. DOI: http://dx.doi.org/10.1590/S000427302013000100004

12. WHO. BMI classification [Internet]. 2006. Disponível em: http://apps.who.int/bmi/index.jsp?introPage=intro_3.html.

13. Heyward VH. Avaliação física e prescrição de exercício: técnicas avançadas. 6. ed. Porto Alegre: Artmed, 2013. xvi, 485 p.

14. Machado, AF. Manual de Avaliação Física. 1 ed. São Paulo: Ícone, 2010.

15. Ling CH, de Craen AJ, Slagboom PE, et al. Accuracy of direct segmental multi-frequency bioimpedance analysis in the assessment of total body and segmental body composition in middle-aged adult population. Clin Nutr 2011;30(5):610-5. DOI: http://dx.doi.org/10.1016/j.clnu.2011.04.001.

16. Dancey CP, Reidy J. Estatística sem matemática para Psicologia. 5. ed. Porto Alegre: Penso, 2013.

17. Fett CA, Fett WCR, Oyama SR, et al. Composição corporal e somatótipo de mulheres com sobrepeso e obesas pré e pós-treinamento em circuito ou caminhada. Rev Bras Med Esporte 2006;12(1):45-50. DOI: http://dx.doi.org/10.1590/S1517-86922006000100009.

18. Andrade Jr, Carlos RM, Clemente EL, et al. Influência da gordura corporal em parâmetros de controle clínico e metabólico de pacientes com diabetes mellitus tipo 1. Arq Bras Endocrinol Metab 2004;48(6):885-889. DOI: http://dx.doi.org/10.1590/S0004$\underline{27302004000600016 .}$ 
19. Rezende FAC, Rosado LEFPL, Franceschinni SCC, et al. Aplicabilidade do índice de massa corporal na avaliação da gordura corporal. Rev Bras Med Esporte 2010;16(2):90-94. DOI: http://dx.doi.org/10.1590/S1517-86922010000200002.

20. Nunes RR, Clemente ELS, Pandini JA, et al. Confiabilidade da classificação do estado nutricional obtida através do IMC e três diferentes métodos de percentual de gordura corporal em pacientes com diabetes melito tipo 1. Arq Bras Endocrinol Metab 2009;53(3):360-367. DOI: http://dx.doi.org/10.1590/S0004-27302009000300011.

21. Neves EB, Ripka WL, Ulbricht L, et al. Comparação do percentual de gordura obtido por bioimpedância, ultrassom e dobras cutâneas em adultos jovens. Rev Bras Med Esporte 2013;19(5):323-327. DOI: http://dx.doi.org/10.1590/S1517-86922013000500004.

22. Nobuyuki M, Akiko T, Miki E, et al. Reference Data of Multi Frequencies Bioelectric Impedance Method in Japanese. Anti-Aging Medicine 2009;6(3):10-14. DOI: http://doi.org/10.3793/jaam.6.10

23. Lee J, Lee JY, Lee JH, et al. Visceral fat obesity is highly associated with primary gout in a metabolically obese but normal weighted population: a case control study. Arthritis Res Ther 2015;17(1):79. DOI: http://dx.doi.org/10.1186/s13075-015-0593-6.

24.Ogawa H, Fujitani K, Tsujinaka T, et al. InBody 720 as a new method of evaluating visceral obesity. Hepatogastroenterology 2011;58(105):42-4.

25. Browning LM, Mugridge O, Chatfield MD, et al. Validity of a new abdominal bioelectrical impedance device to measure abdominal and visceral fat: comparison with MRI. Obesity 2010,18(12):2385-91. DOI: http://dx.doi.org/10.1038/oby.2010.71. 